\title{
Determination of lifetimes of nuclear excited states using the Recoil Distance Doppler Shift Method in combination with magnetic spectrometers
}

\author{
M. Doncel ${ }^{1,2,3, a}$, A. Gadea ${ }^{4}$, J.J. Valiente-Dobón ${ }^{5}$, B. Quintana ${ }^{1}$, V. Modamio ${ }^{5,6}$, D. Mengoni ${ }^{7,8}$, O. Möller ${ }^{9}$, \\ A. Dewald ${ }^{10}$, and N. Pietralla ${ }^{9}$ \\ 1 Laboratorio de Radiaciones Ionizantes, Universidad de Salamanca, Salamanca, Spain \\ 2 Department of Physics, Royal Institute of Technology, SE-10691 Stockholm, Sweden \\ 3 Department of Physics, Oliver Lodge Laboratory, University of Liverpool, Liverpool L69 7ZE, UK \\ 4 Istituto de Física Corpuscular, CSIC-University of Valencia, Valencia, Spain \\ ${ }^{5}$ Laboratori Nazionali di Legnaro, INFN, Legnaro, Italy \\ 6 University of Oslo, Oslo, Norway \\ 7 Dipartimento di Fisica e Astronomia dell'Università di Padova, Padova, Italy \\ 8 Istituto Nazionale di Fisica Nucleare, Sezione di Padova, Padova, Italy \\ 9 Institut für Kernphysik, Technische Universität Darmstadt, Darmstadt, Germany \\ 10 Institut für Kernphysik der Universität zu Köln, Köln, Germany
}

Received: 31 May 2017 / Revised: 4 September 2017

Published online: 27 October 2017

(C) The Author(s) 2017. This article is published with open access at Springerlink.com

Communicated by D. Pierroutsakou

\begin{abstract}
The current work presents the determination of lifetimes of nuclear excited states using the Recoil Distance Doppler Shift Method, in combination with spectrometers for ion identification, normalizing the intensity of the peaks by the ions detected in the spectrometer as a valid technique that produces results comparable to the ones obtained by the conventional shifted-to-unsifted peak ratio method. The technique has been validated using data measured with the $\gamma$-ray array AGATA, the PRISMA spectrometer and the Cologne plunger setup. In this paper a test performed with the AGATA-PRISMA setup at LNL and the advantages of this new approach with respect to the conventional Recoil Distance Doppler Shift Method are discussed.
\end{abstract}

\section{Introduction}

Experimental information on the evolution of nuclear structure is an essential ingredient to shed light on key questions related to nuclear effective interactions. One of the major contemporary topics on nuclear structure is the search for appearance and disappearance of magic numbers revealing the evolution of shell structure towards the drip lines. In this context, knowledge of the electromagnetic transition probabilities, that are very sensitive to the nuclear wave functions, is quite valuable providing evidence of new sub-shell gaps as well as nuclear collectivity. The electromagnetic transition probabilities can be deduced by measuring the lifetimes of the nuclear states that are obtained by using Doppler shift methods, such as the Recoil Distance Doppler Shift method (RDDS). This technique is sensitive to lifetimes of the nuclear excited

\footnotetext{
a e-mail: doncel@liverpool.ac.uk
}

states from a few to hundreds of picoseconds and it has been widely employed in the past by making use of plunger setups in a broad range of nuclear reactions ([1-3] and references therein).

The use of Ge detector arrays coupled with large acceptance magnetic spectrometers allows to measure the $\gamma$ rays in coincidence with the reaction products identified in most cases unequivocally. Such set-ups with outstanding sensitivity have largely contributed to the nuclear structure research during the last decade [4].

A "non-collinear" differential plunger was developed for grazing reactions by the IKP University of Cologne group. This plunger, to be used with a Ge array coupled to a magnetic spectrometer, was commissioned for the first time in a CLARA-PRISMA campaign at the Laboratori Nazionali di Legnaro [4,5] where the CLARA array composed of 25 clover detectors was sucessfully coupled to the large acceptance magnetic spectrometer 
for heavy ions, PRISMA. Several successful measurements were performed with this plunger to determine lifetimes of excited states in neutron-rich nuclei at the CLARAPRISMA setup such as ${ }^{50} \mathrm{Ca}$ and ${ }^{51} \mathrm{Sc}$ isotopes [6] and ${ }^{44,46} \mathrm{Ar}[7]$, as well as for example ${ }^{70^{-74}} \mathrm{Zn}[8]$ or ${ }^{63^{-65}} \mathrm{Co}[9]$ at the AGATA-PRISMA [10] setup. The former measurements were performed during the first physics campaign of the highly segmented AGATA Demonstrator array [11] at LNL. The same plunger was also used with the setup consisting of the EXOGAM gamma spectrometer [12] coupled to the large acceptance magnetic spectrometer VAMOS [13] to measure lifetimes in ${ }^{62,64} \mathrm{Fe}$ [14] and in ${ }^{63,65} \mathrm{Co}[15]$ among others. An application of the differential RDDS technique for "in-flight" relativistic secondary beams has also been developed by the IKP-Cologne group in experiments performed at NSCL with the A1900 separator and S800 spectrometer [16-18]. In such experiments using exotic secondary beams, when Coulomb excitation reactions are used to excite the secondary beam, the $\gamma$-ray emission after the degrader will have contributions from the reactions produced in the degrader itself which may lead to a wrong lifetime determination. So far, the Coulomb inelastic scattering in the degrader has been considered by the implementation of a species selective trigger that suppresses reliably the excitations in the degrader [19] or by using a large distance to determine the yield ratio between the degrader and the target and considering this ratio also as a fit parameter, see refs. $[18,20,21]$.

In the next sections, we will discuss the benefits of the differential Recoil Distance Doppler Shift Method, when the $\gamma$ detectors are used coupled to a magnetic spectrometer, such as PRISMA [5], VAMOS [13], FRS [22,23] or the A1900-S800 [24,25]. As mentioned above, several experiments where identification of the ions has been carried out using magnetic spectrometers have been performed in the latest years at several international accelerator facilities. However, we here report the (to our knowledge) first use of the number of ions recorded by a spectrometer for the lifetime determination and not only to select the nuclei of interest from the reaction products. In this manuscript it will be shown that the measurement in coincidence with the spectrometer might help to solve the problem caused by Coulomb excitation reactions in the degrader. In addittion, nuclei far from stability where the production cross section is low would benefit from the use of only one peak when the lifetime analysis is performed.

\section{The differential Recoil Distance Doppler Shift method with ion identification spectrometers}

The differential RDDS method [1-3] requires a thin target at a fixed position where the nuclide of interest is produced or excited and an energy degrader foil, positioned at a variable distance from the aforementioned target, used to reduce the velocity of the incoming ions which after passing this degrader foil will be eventually identified in a spectrometer. This degrader foil replaces the stopper foil used in the standard plunger technique in which the recoiling nuclei are completely stopped. Two peaks are observed according to the different Doppler shifts depending on where (before or after the degrader) the $\gamma$-ray emission takes place. In this particular case the Doppler correction of the $\gamma$ spectra has been done considering the decays occurring after the degrader (indicated as "unshifted" in the following). The fit of the relative intensities of the two peaks as a function of the target-degrader distance allows to determine the lifetime of the state of interest.

In eq. (1) the intensities of the unshifted and shifted peaks, as a function of the target-degrader distance, corresponding to $\gamma$ rays emitted after and before the degrader, respectively $\left(I_{u}\right.$ and $\left.I_{s}\right)$, are described. $N_{0}$ is the initial population of the state, $d$ is the distance between target and degrader, $v$ is the velocity of the nucleus after leaving the target and $\tau$ is the lifetime of the excited state.

$$
I_{u}(d)=N_{0} e^{\left(\frac{-d}{v \tau}\right)} \quad I_{s}(d)=N_{0}-N_{0} e^{\left(\frac{-d}{v \tau}\right)} .
$$

To obtain an independent value of the number of nuclei produced in the reaction, the decay curve $R$ is defined

$$
R=\frac{I_{u}}{I_{u}+I_{s}} \quad R=e^{\left(\frac{-d}{v \tau}\right)} .
$$

This decay curve corresponds to a simple case of one unique excited state, for a more general form of the decay curve, see ref. [3].

The use of an ion tracking spectrometer, such as PRISMA, where $A$ and $Z$ are determined on an eventby-event basis, allows to develop a different approach to determine the lifetime using only one of the peaks, the shifted or the unshifted one. In this case, the normalization is done considering the number of nuclei populated in the reaction and detected in the spectrometer $\left(N_{I}\right)$. Therefore, the ratio corresponding to the de-excitations "after" $\left(R_{u}\right)$ and "before" $\left(R_{s}\right)$ is obtained as follows:

$$
R_{u}=\frac{I_{u}}{N_{I}}=A e^{\left(\frac{-d}{v \tau}\right)}, \quad R_{s}=\frac{I_{s}}{N_{I}}=A\left(1-e^{\left(\frac{-d}{v \tau}\right)}\right) .
$$

The main difference between the two methods is that in the conventional method one needs to measure accurately the intensity of both peaks to deduce the decay curve as defined in eq. (2) while in the present approach where the number of ions is accurately measured by a spectrometer, the number of useful experimental data increases since two independent normalized decay curves for the shifted and unshifted peaks can be defined, as it can be seen in eq. (3). Therefore, this method can be useful to determine the lifetime of excited states when using a magnetic spectrometer in measurements with low statistics, as expected in future radioactive facilities, or when one of the peaks could not be accurately determined due to low statistics or contaminants from other $\gamma$ transitions. Furthermore, as will be discussed in the following, this normalization of the decay curve with the number of ions $N_{I}$ overcomes the problem of the degrader Coulomb excitations when using the differential RDDS method. 


\section{Measurements with the AGATA $\gamma$-ray array and the PRISMA spectrometer}

The possibility to normalize the $\gamma$-ray transition peaks with the spectrometer data in RDDS experiments has been validated via a measurement performed at the Laboratori Nazionali di Legnaro using the $\gamma$-ray spectrometer AGATA Demonstrator [10,11] coupled to the PRISMA [5] magnetic spectrometer and a compact plunger device, designed and built by the IKP University of Cologne. In particular, we have measured the known lifetime of the $4^{+}$state for the ${ }^{76} \mathrm{Ge}$ isotope [26] and the known lifetimes of the $6^{+}, 4^{+}$and $2^{+}$states for the ${ }^{72} \mathrm{Zn}$ isotope $[8,27,28]$.

To populate the excited states in these nuclei a multinucleon transfer reaction with a ${ }^{76} \mathrm{Ge}$ beam of $577 \mathrm{MeV}$ energy impinging on a ${ }^{238} \mathrm{U}$ target of $1.4 \mathrm{mg} / \mathrm{cm}^{2}$ together with a $1.2 \mathrm{mg} / \mathrm{cm}^{2}$ thick Ta backing has been used. The target was followed by a $4.2 \mathrm{mg} / \mathrm{cm}^{2}$ thickness $\mathrm{Nb}$ degrader foil. The projectile-like reaction products exited the target foil with an average velocity of around $30 \mu \mathrm{m} / \mathrm{ps}(v / c=10 \%)$. The $\mathrm{Nb}$ foil degraded the energy of the ions and resulted in an average velocity of $27 \mu \mathrm{m} / \mathrm{ps}(v / c=9 \%)$. The projectile-like reaction nuclei were detected and identified in the magnetic spectrometer PRISMA, which was positioned at the grazing angle with respect to the beam axis $\left(55^{\circ}\right)$. Using PRISMA the mass, atomic number and velocity of the different ions are measured providing a clean selectivity of the different channels and an accurate Doppler correction. Emitted $\gamma$ rays were detected with the AGATA Demonstrator located at backward angles where a higher sensitivity for Doppler lifetime measurements is achieved. Only four, of the five clusters of the AGATA Demonstrator, were available. The detectors were placed at $18.1 \mathrm{~cm}$ from the target position and the peak efficiency of the detection system was $3.2 \%$ at $1.3 \mathrm{MeV}$. In order to cover a range from approximately 2 to $30 \mathrm{ps}$, measurements were taken for five different targetdegrader distances: $112 \mu \mathrm{m}, 212 \mu \mathrm{m}, 512 \mu \mathrm{m}, 1012 \mu \mathrm{m}$ and $1912 \mu \mathrm{m}$.

\subsection{Lifetime determination of the $4^{+}$state in ${ }^{76} \mathrm{Ge}$}

The inelastic channel of the ${ }^{76} \mathrm{Ge}$ beam $+{ }^{238} \mathrm{U}$ target reaction has been used to prove that the normalization with the spectrometer overcomes the problem of Coulomb excitations in the degrader since inelastic excitation can take place in both the target and the degrader foils. As usually the thickness of the degrader is significantly larger than the target one, the yield production in the degrader is not negligible.

For this analysis, the $4^{+} \rightarrow 2^{+}$transition at $847 \mathrm{keV}$ energy in the ${ }^{76} \mathrm{Ge}$ isotope has been used since large statistics and no contaminants were present, see fig. 1. The lifetime of the $4^{+}$state is known to be 2.6(6) ps [26]. In this case the lifetime could be determined by fitting a simple exponential decay curve, since no feeding to the $4^{+}$state has been observed in the present experiment.

As performed in previous works $[18,19]$, long distance measurements $(1012 \mu \mathrm{m}$ and $1912 \mu \mathrm{m})$ have been used to

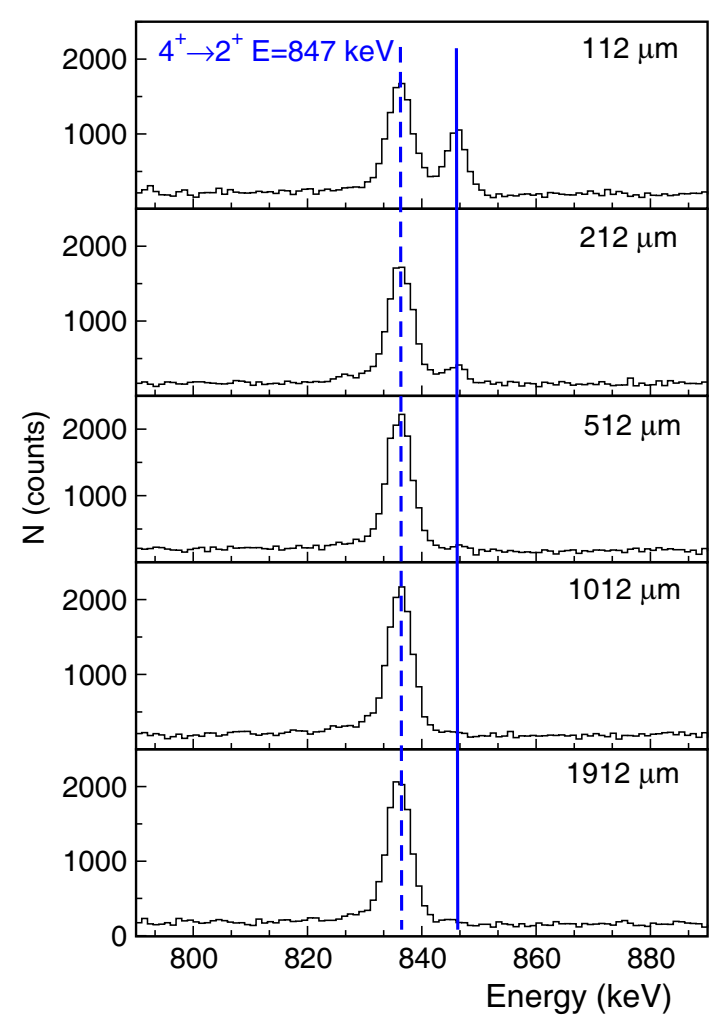

Fig. 1. Doppler-corrected $\gamma$-ray spectra for ${ }^{76} \mathrm{Ge}$ in the region of interest, around the $4^{+} \rightarrow 2^{+}$transition at $847 \mathrm{keV}$, for the different distances. The solid line plotted corresponds to the unshifted peak and the dashed line to the shifted peak.

extract the contribution coming from the inelastic scattering in the degrader. The contribution from the degrader is $2(1) \%$ of the one from the shifted peak. Therefore, in this case, the contribution of the inelastic scattering of the degrader is not negligible. For the shifted peak approach the decay curve from eq. (3) has been employed while for the unshifted peak a constant value to take into account the uniform contribution of the Coulomb excitation produced in the degrader has been added to the fitting function. Therefore, with this new method there is no need for a long distance measurement to quantify the amount of inelastic scattering produced in the degrader. This represents an important reduction of the required beam time during the experiment.

Figure 2 shows the various fits of the corresponding decay function performed for the different decay curves. table 1 shows the $\tau$ values for the $4^{+}$state determined after the fitting procedure using the different decay curves. The results are denoted in the table as conventional (eq. (2)), unshifted and shifted. The reduced transition probabilities have been included for completeness.

The agreement between the literature value and the ones obtained in this work allows to confirm that this normalization technique can be successfully used for lifetime determination when the RDDS method is combined with an ion identification spectrometer. 

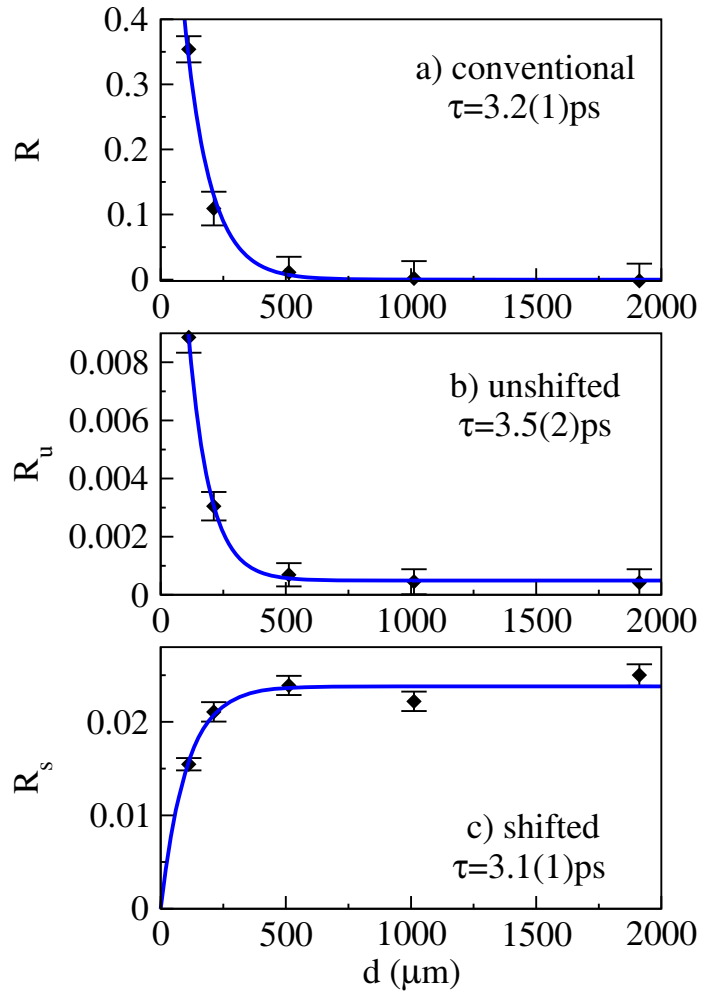

Fig. 2. Fitted exponential decay curve for the $4^{+} \rightarrow 2^{+}$transition at $847 \mathrm{keV}$ in ${ }^{76} \mathrm{Ge}$ considering the following scenarios: (a) when the conventional method is applied, (b) when only the unshifted peak is used and normalized with $N_{I}$ and (c) when only the shifted peak is used and normalized with $N_{I}$.

Table 1. Lifetime $\tau$ and reduced transition probability $B(E 2 \downarrow)$ for the $4^{+} \rightarrow 2^{+}{ }^{76} \mathrm{Ge}$ transition at $847 \mathrm{keV}$ measured with the different approaches presented in this work. The literature value is taken from ref. [26].

\begin{tabular}{c|c|c}
\hline Method & $\tau(\mathrm{ps})$ & $B(E 2 \downarrow)\left(\mathrm{e}^{2} \mathrm{fm}^{4}\right)$ \\
\hline literature & $2.6(6)$ & $722(167)$ \\
conventional & $3.2(1)$ & $588(18)$ \\
unshifted & $3.5(2)$ & $537(31)$ \\
shifted & $3.1(1)$ & $607(20)$ \\
\hline
\end{tabular}

\subsection{Lifetime determination of the first excited states in the ${ }^{72} \mathrm{Zn}$ isotope}

In order to further validate these RDDS measurements, based on the normalization to the number of detected ions, the lifetime analysis has also been performed for ${ }^{72} \mathrm{Zn}$, populated via a $2 \mathrm{p} 2 \mathrm{n}$ multi-nucleon transfer reaction. In particular, the $2^{+} \rightarrow 0^{+}$transition at $653 \mathrm{keV}$ has been studied considering the feeding coming from the $6^{+} \rightarrow 4^{+}$and $4^{+} \rightarrow 2^{+}$transitions at 1154 and $847 \mathrm{keV}$, respectively.

The results obtained in this section are compared with the literature values obtained when the Decay Curve method is applied to the same experimental data [8] and with the previous measurements done by Leenhardt et al. [27] and by Niikura et al. [28]. The determination of
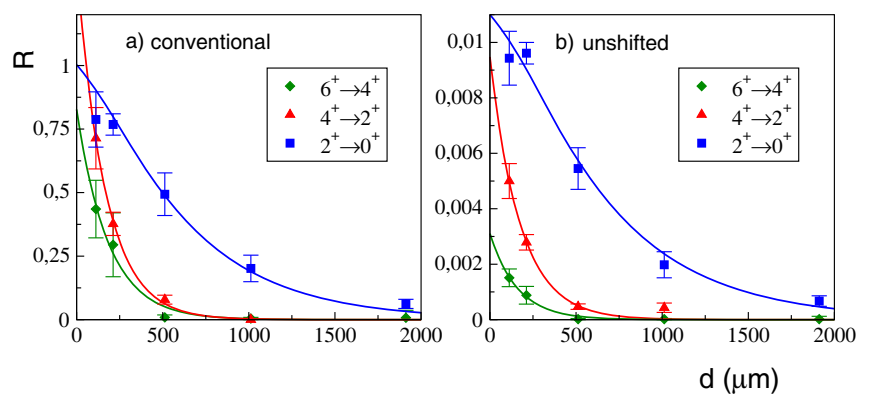

Fig. 3. Fitted decay curves for the $6^{+} \rightarrow 4^{+}, 4^{+} \rightarrow 2^{+}$and $2^{+} \rightarrow 0^{+}$transitions in the ${ }^{72} \mathrm{Zn}$ isotope at 1154,847 and $653 \mathrm{keV}$, respectively, (a) when the conventional method is applied and (b) when the unshifted peak is considered normalized with the number of ions $N_{I}$.

the lifetime is done through a decay curve fit as defined in eq. (6) in ref. [3] that considers the highest feeding levels, that for the case of the $2^{+}$, corresponds to the $6^{+}$and $4^{+}$states. The decay curve fits for the three states $\left(2^{+}\right.$, $4^{+}$and $6^{+}$) are shown in fig. 3 for the conventional as well as for the present approach using the unshifted peak. In this case, the new approach considering the shifted peak has not been employed since not enough distances were measured to determine the asymptotic line of the exponential decay.

The values obtained for the lifetime and the reduced transition probability of the $6^{+} \rightarrow 4^{+}, 4^{+} \rightarrow 2^{+}$and $2^{+} \rightarrow 0^{+}$transitions at 1154,847 and $653 \mathrm{keV}$, respectively, obtained with both RDDS approaches are summarized in table 2 . As for ${ }^{76} \mathrm{Ge}$, there is a good agreement between the lifetime literature values and the values measured in this work with both RDDS normalizations. As it is shown in tables 1 and 2, the precision of the method applied in this work is comparable with the one obtained when the conventional method is employed. This can be understood since the main contribution to the lifetime uncertainty is coming from the uncertainty in the number of counts of each peak, which is the same in both methods. This further confirms that the normalization to the number of ions is a promising method for future use with radioactive ion beams.

\section{Conclusions}

The lifetime of excited states in nuclei has been measured using the Recoil Distance Doppler Shift method together with a spectrometer for ion identification. The coincidence measurement allows to determine lifetimes using only one of the peaks (due to emission before or after the degrader) for each $\gamma$-ray transition. The present method has been validated for some excited states in the ${ }^{72} \mathrm{Zn}$ and ${ }^{76} \mathrm{Ge}$ isotopes showing a good agreement with the conventional known values.

One advantage of this normalization is the possibility to determine the lifetime for nuclei far from stability where the experimental accessibility is difficult and, therefore, the statistics is marginal. In this case only one peak can be enough to determine the lifetime of the excited 
Table 2. Lifetime $\tau$ and reduced transition probability $B(E 2 \downarrow)$ for the $6^{+} \rightarrow 4^{+}, 4^{+} \rightarrow 2^{+}$and $2^{+} \rightarrow 0^{+}$transitions at 1154,847 and $653 \mathrm{keV}$, respectively, obtained with the different approaches in this work. The literature values are taken from refs. $[8,27,28]$.

\begin{tabular}{c|c|c|c|c|c|c}
\hline & \multicolumn{2}{|c}{$6^{+} \rightarrow 4^{+}$} & \multicolumn{3}{c}{$4^{+} \rightarrow 2^{+}$} & $2^{+} \rightarrow 0^{+}$ \\
\hline Method & $\tau(\mathrm{ps})$ & $B(E 2 \downarrow)\left(\mathrm{e}^{2} \mathrm{fm}^{4}\right)$ & $\tau(\mathrm{ps})$ & $B(E 2 \downarrow)\left(\mathrm{e}^{2} \mathrm{fm}^{4}\right)$ & $\tau(\mathrm{ps})$ & $B(E 2 \downarrow)\left(\mathrm{e}^{2} \mathrm{fm}^{4}\right)$ \\
\hline literature & $3.0(9)$ & $134_{-31}^{+57}$ & $5.2_{-0.7}^{+0.8}$ & $361_{-47}^{+57}$ & $17.6(14)[8]$ & $392_{-29}^{+34}$ \\
& & & & & $19.8(24)[27]$ & $348(42)$ \\
& & & & & $17.9(18)[28]$ & $385(39)$ \\
conventional & $3.6(4)$ & $111(12)$ & $4.9(5)$ & $383(40)$ & $19(2)$ & $371(36)$ \\
unshifted & $3.3(6)$ & $121(22)$ & $4.8(6)$ & $391(49)$ & $18(2)$ & $383(43)$ \\
\hline
\end{tabular}

state of interest. A second advantage is associated with the determination of the different contributions coming from the Coulomb excitation of a radioactive ion either from the target or the degrader. This normalization to the spectrometer data has solved this problem in the case of AGATA-PRISMA data, and it could avoid extra measurements at very large target-degrader distances. These advantages might be relevant in a time in which radioactive ion beam facilities are being developed to produce very exotic beams further away from the stability.

This work has been partially supported by MINECO, Spain, under grants AIC-D-2011-0746 and FPA2011-29854, by the Generalitat Valenciana, Spain, under grant PROMETEO/2010/101 and by the Swedish Research Council under grant No. 621-2010-3694.

Open Access This is an open access article distributed under the terms of the Creative Commons Attribution License (http://creativecommons.org/licenses/by/4.0), which permits unrestricted use, distribution, and reproduction in any medium, provided the original work is properly cited.

\section{References}

1. T.K. Alexander, J.S. Foster, Adv. Nucl. Phys. 10, 197 (1978).

2. A. Dewald, S. Harissopulos, P. Brentano, Z. Phys. A 334, 163 (1989).

3. A. Dewald et al., Prog. Part. Nucl. Phys. 67, 786 (2012).
4. A. Gadea et al., Acta. Phys. Pol. B 38, 1311 (2007).

5. S. Silzner et al., Phys. Rev. C 76, 024604 (2007).

6. J.J. Valiente-Dobón et al., Phys. Rev. Lett. 102, 242502 (2009).

7. D. Mengoni et al., Phys. Rev. C 82, 024308 (2010).

8. C. Louchart et al., Phys. Rev. C 87, 054302 (2013).

9. V. Modamio et al., Phys. Rev. C 88, 044326 (2013).

10. A. Gadea et al., Nucl. Instrum. Methods A 654, 88 (2011).

11. S. Akkoyun et al., Nucl. Instrum. Methods A 668, 26 (2012).

12. S.L. Shepherd et al., Nucl. Instrum. Methods A 434, 373 (1999).

13. S. Pullanhiotan et al., Nucl. Instrum. Methods A 593, 343 (2008).

14. J. Ljungvall et al., Phys. Rev. C 81, 061301 (2010).

15. A. Dijon et al., Phys. Rev. C 83, 064321 (2011).

16. A. Dewald et al., Phys. Rev. C 78, 051302(R) (2008).

17. K. Starosta et al., Phys. Rev. Lett. 99, 042503 (2007).

18. W. Rother et al., Phys. Rev. Lett. 106, 022502 (2011).

19. W. Rother et al., Nucl. Instrum. Methods A 654, 196 (2011).

20. P. Voss et al., Phys. Rev. C 86, 011303 (2012).

21. M. Petri et al., Phys. Rev. C 86, 044329 (2012).

22. H. Geissel et al., Nucl. Instrum. Methods B 70, 286 (1992).

23. H. Geissel et al., Annu. Rev. Nucl. Part. Sci. 45, 163 (1995).

24. D.J. Morrissey et al., Nucl. Instrum. Methods B 204, 90 (2003).

25. D. Bazin et al., Nucl. Instrum. Methods B 204, 29 (2003).

26. R. Lecomte et al., Phys. Rev. C 22, 2420 (1980).

27. S. Leenhardt et al., Eur. Phys. J. A 14, 1 (2002).

28. M. Niikura et al., Phys. Rev. C 85, 054321 (2012). 